OPEN ACCESS

Edited by:

Daniela Merlotti,

University of Siena, Italy

Reviewed by:

Zhi Xiang Ng,

University of Nottingham Malaysia

Campus, Malaysia

Michael Edwin Edmonds,

King's College Hospital NHS

Foundation Trust, United Kingdom

*Correspondence: Mattabhorn Phimphilai mphimphi@hotmail.com

Specialty section: This article was submitted to Bone Research, a section of the journal

Frontiers in Endocrinology

Received: 22 October 2021 Accepted: 18 January 2022

Published: 14 February 2022

Citation:

Phimphilai M, Pothacharoen P, Chattipakorn $N$ and Kongtawelert $P$

(2022) Receptors of Advanced

Glycation End Product (RAGE)

Suppression Associated With a

Preserved Osteogenic Differentiation in

Patients With Prediabetes.

Front. Endocrinol. 13:799872. doi: 10.3389/fendo.2022.799872

\section{Receptors of Advanced Glycation End Product (RAGE) Suppression Associated With a Preserved Osteogenic Differentiation in Patients With Prediabetes}

\author{
Mattabhorn Phimphilai ${ }^{1 *}$, Peraphan Pothacharoen ${ }^{2}$, Nipon Chattipakorn ${ }^{3,4}$ \\ and Prachya Kongtawelert ${ }^{2}$
}

1 Division of Endocrinology, Department of Internal Medicine, Faculty of Medicine, Chiang Mai University, Chiang Mai, Thailand, 2 Thailand Excellence Center for Tissue Engineering and Stem Cells, Department of Biochemistry, Faculty of Medicine, Chiang Mai University, Chiang Mai, Thailand, ${ }^{3}$ Cardiac Electrophysiology Research and Training Center, Faculty of Medicine, Chiang Mai University, Chiang Mai, Thailand, ${ }^{4}$ Department of Physiology, Faculty of Medicine, Chiang Mai University, Chiang Mai, Thailand

Type 2 diabetes is widely documented for osteogenic differentiation defect and impaired bone quality, which is related to the skeletal accumulation of advanced glycation end products (AGEs). Prediabetes is a condition in which hyperglycemia is lower than the threshold for the diagnosis of diabetes. Prediabetic animal models consistently demonstrate impaired osteogenic differentiation and deteriorated bone microarchitecture. However, no evidence shows defects in osteoblast development and skeletal effects of AGEs in prediabetic individuals. Therefore, it remains to be elucidated whether impaired osteogenic differentiation ability and altered cellular response to AGEs occur in patients with prediabetes. This cross-sectional study included 28 patients with prediabetes as defined by impaired fasting glucose criteria, fasting plasma glucose (FPG) between $100-125 \mathrm{mg} / \mathrm{dl}$ and 17 age-matched normoglycemic controls to elucidate osteogenic differentiation and AGER expression in the PBMC derived from those individuals. The PBMC-isolated from both groups showed similar rates of expression of osteoblast-specific genes, namely, ALPL, BGLAP, COL1A1, and RUNX2/PPAR (89.3\% and 88.2\%, $p=1.000$ ), and showed comparable levels of expression of those genes. By using age- and pentosidine-matched normoglycemic individuals as references, the PBMC-isolated from prediabetic patients demonstrated lower expression of both $A G E R$ and BAX/BCL2. The expression of AGER and BAX/BCL2 significantly correlated to each other $(r=0.986, p<0.0001)$. The multivariate analysis demonstrated that serum pentosidine is an independent risk factor for AGER expression. With logistic regression analysis, the area under the ROC curve (AUC) for serum pentosidine at the cut-off level of $2.1 \mathrm{ng} / \mathrm{ml}$ and FPG at $100 \mathrm{mg} / \mathrm{dll}$, which is a cut-off 
point for prediabetes, was significantly higher for predicting AGER expression than that of serum pentosidine alone (0.803 vs $0.688, p=0.048$ ), indicating that serum pentosidine was a good predictor of $A G E R$ expression in prediabetic individuals. In conclusion, this study demonstrated a preserved osteogenic differentiation in the PBMC derived from prediabetic individuals. In addition, those PBMC with preserved osteogenic differentiation potential showed the suppression of both cellular RAGE and apoptotic-related signals. Serum pentosidine was an independent risk factor for cellular RAGE expression and is conceivably a good predictor for AGER suppression in prediabetic individuals.

Keywords: advanced glycation end products, impaired fasting glucose, osteogenic differentiation, prediabetes, peripheral blood mononuclear cell, receptor of advanced glycation end products, type 2 diabetes

\section{INTRODUCTION}

Type 2 diabetes is a health problem of great concern worldwide. It is a metabolic disorder that has insulin resistance as a central pathophysiology. That insulin resistance occurs many years before the development of progressive dysglycemia, starting from modest hyperglycemia in prediabetes to full-blown chronic hyperglycemia in type 2 diabetes. According to the American Diabetes Association, prediabetes can be diagnosed by one of the three following criteria (1): first, impaired fasting glucose (IFG) defined by fasting plasma glucose (FPG) of between 100 and $125 \mathrm{mg} / \mathrm{dl}$; second, impaired glucose tolerance (IGT) defined by FPG of between 140 and $199 \mathrm{mg} / \mathrm{dl}$ at $2 \mathrm{~h}$ after $75 \mathrm{~g}$ of oral glucose loading; and third, a level of glycated hemoglobin (HbAlc) that is between 5.7 and 6.4\% (1). It is well documented that chronic hyperglycemia accelerated the accumulation of advanced glycation end products (AGEs) in many tissues, influencing the occurrence of chronic microvascular and macrovascular complications found in patients with diabetes $(2-5)$. Even though prediabetes is a condition with hyperglycemia which is lower than the threshold for the diagnosis of diabetes, prediabetes can also lead to similar chronic microvascular and macrovascular complications to those found in individuals with diabetes (6, 7). However, there is no evidence to show the association between the accumulation of AGEs and those complications found in prediabetes. Moreover, Gateva and colleagues (8) demonstrated that serum pentosidine, a type of AGEs, did not relate to vascular complications in prediabetic individuals.

Type 2 diabetic individuals consistently showed an impaired bone quality represented by a decreased bone turnover $(9,10)$, deteriorated bone microarchitecture (11), and an increased risk of fragility fractures (12-15) even though they had a preserved bone mineral density (13-15). Individuals with prediabetes who have a milder level of hyperglycemia than those with diabetes were also shown for low bone turnover $(16,17)$ and preserved bone mineral density (17-20). However, evidence involving impairment of bone quality and fragility fractures in prediabetic individuals is still controversial. Chen and colleagues (19) and Park and colleagues (21) demonstrated an increased risk of hip fractures in a prediabetic population. In contrast, Dominic and colleagues (22), and Iki and colleagues (23), demonstrated that hip fractures did not increase in a population with prediabetes. In addition, Dowson-Hughes and colleagues (24) showed the preservation of bone material strength in individuals with prediabetes.

AGEs exert their downstream signaling cascades, including inflammatory signaling pathways and apoptotic pathway, via interacting with their specific receptors, the receptor of advanced glycation end product (RAGE) (25). The polymorphism of the RAGE gene is associated with proinflammation and oxidative stress, and also with diabetic retinopathy, a microvascular complication of diabetes (26). The activation of RAGE lead to dysfunction and apoptosis of cells, namely, osteogenic lineage cells (27-32). The accumulation of AGEs conceivably contributes to the impairment of bone quality found in type 2 diabetes. Preclinical studies have shown that the skeletal accumulation of AGEs inhibited osteoblast differentiation (28-30), enhanced osteoblast apoptosis $(27,29-31)$, and deteriorated the mechanical properties of the skeleton (33-35). In human subjects, our previous studies showed that the peripheral blood mononuclear cells (PBMC) derived from patients with type 2 diabetes had an impaired osteogenic differentiation potential which could be linked to the overexpression of $\operatorname{RAGE}(36,37)$. In addition, serum pentosidine has been shown to have a positive correlation with vertebral fractures in patients with type 2 diabetes (38-40). Although AGEs have been widely documented for their detrimental effects on bone cells and bone strength in type 2 diabetes, the effects of AGEs on the skeleton remain inconclusive in prediabetes. To date, there have only been a few preclinical studies that demonstrated an adverse effect of being prediabetic on osteoblast function and the skeletal microarchitecture. Pramojanee and colleagues (41) showed a decrease in osteoblast proliferation and survival, and a deterioration of bone microarchitecture in prediabetic rats. In addition, Ross and colleagues (42) demonstrated a decreased osteoblast differentiation and compromised cortical bone microarchitecture in prediabetic mice. However, both studies did not explore the effects of AGEs on those cellular dysfunction and skeletal microarchitecture deterioration. To date, there is no evidence to show osteoblast dysfunction and the skeletal impacts of AGEs in patients with prediabetes. Therefore, it remains to be elucidated whether an impairment of osteogenic differentiation or an alteration of cellular RAGE expression occurs in prediabetic patients. 
Mesenchymal stem cells can be derived from various adult tissues including adipose tissue, bone marrow and peripheral blood (43). It is well documented that the peripheral bloodderived mesenchymal stem cells (PB-MSC) can differentiate into multiple cell types, namely, adipocytes, chondrocytes, and osteoblasts (44-46). In terms of osteoblast differentiation, Valenti and colleagues (47) demonstrated the expression of multiple osteoblast-specific genes during differentiation of the PB-MSC, namely, COL1A1 and RUNX2, and also the production of the $B G A L P$-encoded protein named osteocalcin. In addition, our previous studies also demonstrated the differentiation toward osteoblast of the PBMC-isolated from both nondiabetic and diabetic individuals $(36,37)$. Therefore, to obtain those stem cells with the least invasive measure, this study was conducted using the PBMC-isolated from participants to investigate the osteogenic differentiation potential of the stem cells. This study aimed to determine whether 1) there was an osteogenic differentiation defect in PBMC derived from prediabetic individuals, and 2) there was an alteration of RAGE expression in PBMC isolated from patients with prediabetes.

\section{MATERIALS AND METHODS}

\section{Ethics Statement}

This study was a cross-sectional study, performed at the Maharaj Nakorn Chiang Mai Hospital, Chiang Mai University, Chiang Mai, Thailand and approved by the Research Ethics Committee of the Faculty of Medicine, Chiang Mai University. All participants provided their written informed consent to participate in this study before enrollment.

\section{Study Population and Sample Collection}

Individuals with FPG between 100 and $125 \mathrm{mg} / \mathrm{dl}$ on at least two occasions, which were classified as impaired fasting glucose (IFG) by the American Diabetes Association, were enrolled as prediabetic subjects. Age-matched individuals with FPG less than $100 \mathrm{mg} / \mathrm{dl}$ were enrolled as normoglycemic subjects. Individuals with IFG were excluded if $\mathrm{HbAlc}$ higher than $6.4 \%$. The other exclusion criteria were as follows: females with serum creatinine higher than $1.4 \mathrm{mg} / \mathrm{dl}$ males with serum creatinine above $1.5 \mathrm{mg} / \mathrm{dl}$; individuals who use steroids, antiresorptive agents or anabolic agents for osteoporosis, immunosuppressive agents, thiazolidinedione; and individuals with hematologic or metastatic malignancy. Venous blood (35-40 ml) was collected from all enrolled participants to isolate the PBMC, and to determine serum levels of pentosidine (Elabscience Biotechnology, WuHan, Hubei, China), soluble RAGE (sRAGE) (R\&D, Minneapolis, MN, USA), interleukin 1- $\beta$ (IL1- $\beta$ ) (R\&D, Minneapolis, MN, USA) and tumor necrosis factor- $\alpha$ (TNF- $\alpha)(\mathrm{R} \& \mathrm{D}$, Minneapolis, $\mathrm{MN}$, USA) by ELISA. FPG, HbA1c, serum creatinine, high-density lipoprotein cholesterol (HDL-C), low-density lipoprotein cholesterol (LDL-C), and triglyceride levels were assessed using standardized procedures at the central laboratory of the Faculty of Medicine, Chiang Mai University. Glomerular filtration rate (eGFR) was calculated using the Chronic Kidney Disease Epidemiology Collaboration (CKD-EPI) method. Fracture risk estimation was estimated from the Fracture Risk Assessment Tool (FRAX ${ }^{\circledR}$ ) using the Thailand database (48).

\section{Human Peripheral Blood-Derived Mononuclear Cells (PBMC) Isolation and Culture Protocol}

Peripheral venous blood $(35-40 \mathrm{ml})$ was applied to density gradient centrifugation and PBMC were isolated and then cultured as described in our previous study (36). In brief, the plasma was removed from the venous blood by centrifugation at 1,500 rpm for $5 \mathrm{~min}$. The remaining cell fraction was first diluted with an equal volume of DMEM (Gibco, Grand Islands, NY, USA) and then overlaid on Histopaque (specific gravity $1.077 \mathrm{~g} /$ $\mathrm{ml}$; Sigma-Aldrich, St. Louis, MO, USA) and further centrifuged at 4,000 rpm for $30 \mathrm{~min}$. The cells in the mononuclear cell layer (PBMC) were plated in 24-well culture plates and cultured in RPMI supplemented with $10 \%(\mathrm{v} / \mathrm{v})$ fetal bovine serum (Gibco, Grand Islands, NY, USA). The floating cells in the culture wells were removed and the plastic-adhered cells were further cultured in a non-osteogenic-inducing medium, DMEM supplemented with $10 \%$ (v/v) fetal bovine serum (Gibco, Grand Islands, NY, USA), for 7-10 days until confluence. To induce osteogenic differentiation, the adhered cells were cultured in a nonosteogenic-inducing medium until reaching 50\% confluence. They were then transferred to an osteogenic-inducing medium; DMEM supplemented with $10^{-7} \mathrm{M}$ dexamethasone, $60 \mu \mathrm{M}$ ascorbic acid, and $10 \mathrm{mM} \beta$-glycerophosphate) and cultured for a further 21 days.

\section{Analysis of the Expression of Osteoblast- Specific Genes, AGER, and Cellular Apoptotic-Associated Genes}

The expression of genes, including osteoblast-specific genes, $A G E R, B A X$, and $B C L 2$, was quantified using real-time PCR as described in our previous study $(36,37)$. In brief, the total RNA (500 ng) was isolated from the cell lysate using the illutraRNA spin Mini Kit (GE Healthcare Life Science, Buckinghamshire/ Little Chalfont, UK) following the manufacturer's instructions. The isolated total RNA of each sample was reverse transcribed into cDNA using an iScript ${ }^{\mathrm{TM}}$ CDNA Synthesis Kit (Bio-Rad, Hercules, CA, USA) following the manufacturer's protocol. The CDNA was subsequently analyzed by reversed transcription PCR (RT-PCR) using Sso7d fusion enzyme technology according to the manufacturer's instruction (Bio-Rad, Hercules, CA, USA). The PCR protocol consisted of 45 cycles of $5 \mathrm{~s}$ at $95^{\circ} \mathrm{C}, 10 \mathrm{~s}$ at $60^{\circ} \mathrm{C}$, and $30 \mathrm{~s}$ at $72^{\circ} \mathrm{C}$ using the Applied Biosystems $7500 / 7500$ Fast Real-Time PCR system. The total RNA isolated from both non-osteogenic and osteogenic-inducing cells was used to analyze: 1) osteoblast-specific genes, namely, $A L P L, B G L A P$, $C O L 1 A 1$, and RUNX2 for representing osteoblast differentiation and 2) PPAR- $\gamma$ which is a transcription factor driving towards adipocytes for evaluating signals against osteoblast 
differentiation. In this study, since multiple osteoblast-specific genes, including $A L P L, B G L A P, C O L 1 A 1$, and $R U N X 2$, were expressed during the differentiation process toward osteoblasts $(47,49)$ and were persistently elevated in human osteoblasts $(49,50)$, the differentiation towards osteoblasts was defined by the increment of expression of all osteoblast-specific marker genes, including $A L P L, B G L A P$, and $C O L 1 A 1$, and also the increment of the $R U N X 2 / P P A R \gamma$ ratio. In contrast, the total RNA extracted only from the non-osteogenic-inducing cells was used to determine: 1) AGER expression to elucidate the alteration of cellular RAGE expression and 2) BAX and BCL2 expression for evaluation of cellular apoptotic signals. The GAPDH expression was used for normalization of the relative expression levels for each primer set (Table 1) by the $2^{(-\Delta \Delta \mathrm{CT})}$ method. All primers were purchased from Invitrogen.

\section{Statistical Analysis}

Statistical analysis was performed using SPSS version 23.0.All continuous data are reported as mean \pm standard deviation while all categorical variables are presented as percentages. An independent $t$-test was used for univariable comparative statistics for continuous data. A Chi-square test was used for univariable comparative statistics for all categorical variables, the exception being the categorical variable with small counts which was analyzed using Fisher's Exact test. Pearson's correlation was performed to identify the correlation between two continuous variables. Multivariate linear regression analysis was performed to identify the independent risk factors of AGER expression. A logistic regression model was performed to determine the potential for using serum pentosidine as a predictor of $A G E R$ suppression in prediabetic individuals. The areas under the receiver operating characteristics (ROC) curves of the model (AUC) were plotted to determine the diagnostic performance of the serum pentosidine cut-off value which was calculated based on the highest sensitivity or specificity. A p-value of less than 0.05 was used as a measure of statistical significance. A sample size calculation was performed to estimate the number needed to show the non-inferiority of osteogenic differentiation in the prediabetic group compared to the normoglycemic group (51).
A sample size of at least $12-16$ participants in the normoglycemic group and 17-23 patients in the prediabetic group was estimated to give $80 \%$ power at the $5 \%$ significance level to detect a non-inferiority of osteogenic differentiation in the prediabetic group compared to the normoglycemic group at a margin of equivalence of $20-25 \%(36,51)$.

\section{RESULTS}

\section{Demographic Data, Clinical Characters, and Biochemical Parameters of Study Participants}

This study included 28 individuals with prediabetes and 17 agematched participants with normoglycemia. All participants with prediabetes were diagnosed with impaired fasting glucose (IFG) using the FPG criteria with a cut-off value of $100-125 \mathrm{mg} / \mathrm{dl}$ as recommended by the American Diabetes Association (1). All prediabetic participants had at least two FPG instances between 100 and $125 \mathrm{mg} / \mathrm{dl}$ during the three months before or after the recruitment date. All participants in the normoglycemic group had never had a recorded FPG higher than $99 \mathrm{mg} / \mathrm{dl}$ before enrollment. Age, gender, body mass index (BMI), systolic blood pressure (SBP), diastolic blood pressure (DBP), eGFR, HDL-C, LDL-C, triglyceride and 10-year fracture risk as determined by FRAX $^{\circledR}$ using the Thailand database were comparable in both normoglycemic and prediabetic groups (Table 2). Either angiotensin-converting enzyme inhibitors (ACEI) or angiotensin II receptor blockers (ARB) were used at a higher rate in patients with prediabetes while dihydropyridine calcium channel blocker (DHP-CCB) was used at a higher rate in participants with normoglycemia; however, the difference did not reach statistical significance (Table 2). Lipid-lowering agents, namely, statins and fibrates were used at a comparable rate in both normoglycemic and prediabetic groups (Table 2).

In the group with prediabetes, the duration of being prediabetic was $3.4 \pm 2.8$ years. Patients within the prediabetic group had a significantly higher level of FPG than those in the normoglycemic group $(102.1 \pm 10.7 \mathrm{mg} / \mathrm{dl}$ vs $90.2 \pm 6.3 \mathrm{mg} / \mathrm{dl}$,

TABLE 1 | Sequences of Real-Time qPCR Primers.

\begin{tabular}{|c|c|c|}
\hline Genes & \multicolumn{2}{|c|}{ Primer sequence $\left(5^{\prime}-3^{\prime}\right)$} \\
\hline$A L P L$ & CATGGCTTGGGCAGAAGGA & CTAGCCCCAAAAAGAGTTGCAA \\
\hline$B G L A P$ & GAAGCCCAGCGGTGCA & САСТАССТСGСТGСССТСС \\
\hline COL1A1 & CAGCCGCTTCACCTACAGC & ПTाGTATTCAATCACTGTCTTGCC \\
\hline RUNX2 & TCTTAGAACAAATTCTGCCCTाT & TGCTTTGGTCTTGAAATCACA \\
\hline \multicolumn{3}{|c|}{ Apoptotic signal-associated genes } \\
\hline$B A X$ & TGGAGCTGCAGAGGATGATTG & GAAGTTGCCGTCAGAAAACATG \\
\hline BCL2 & CATGCTGGGGCCGTACAG & GAA CCGGCACCTGCACAC \\
\hline \multicolumn{3}{|l|}{ Others } \\
\hline AGER & GCTGGAATGGAAACTGAACACAGG & TTCCCAGGAATCTGGTAGACACG \\
\hline GAPDH & СССТTСАТGАССТСААСТА & AGATGATGACCCTITGGCT \\
\hline
\end{tabular}


TABLE 2 | Clinical Characteristics of the Study Participants.

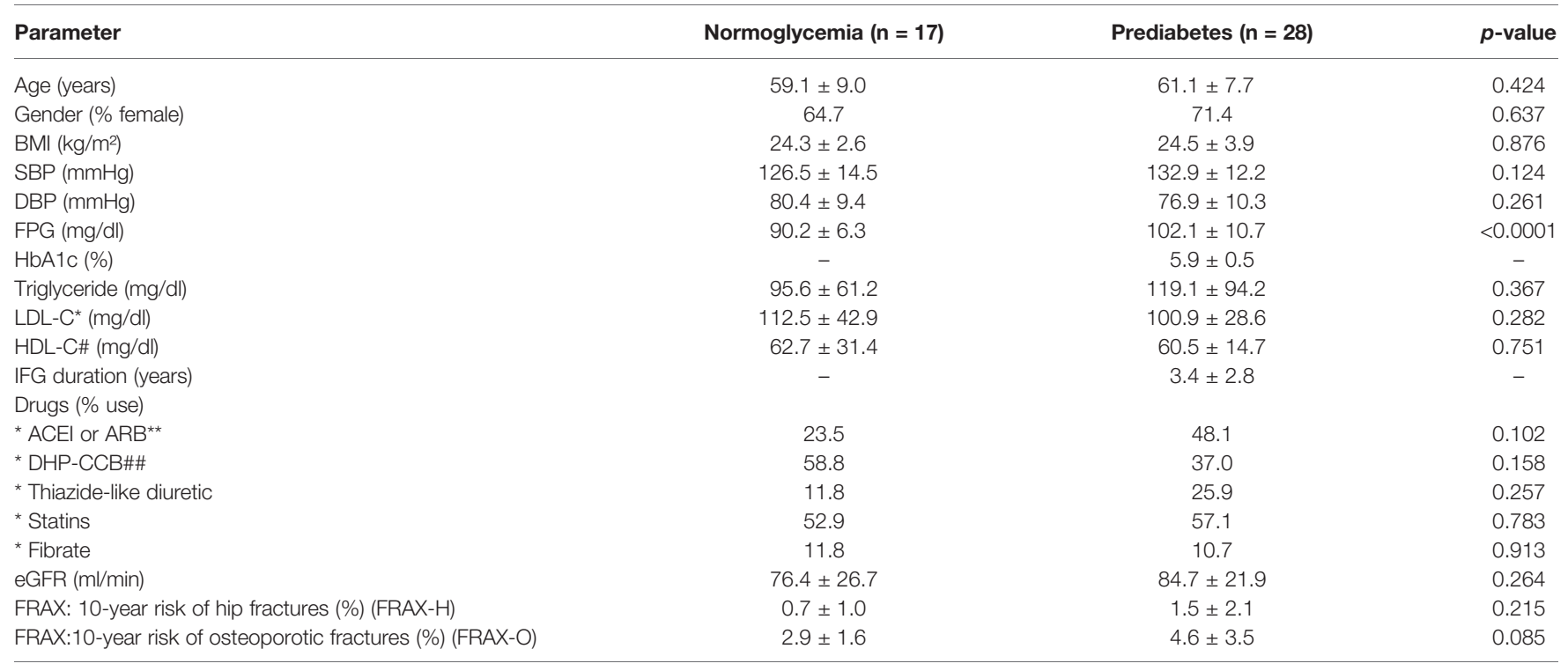

*LDL-C, low-density lipoprotein cholesterol.

"HDL-C, high-density lipoprotein cholesterol.

${ }^{*} A C E l$ angiotensin-converting enzyme inhibitors.

${ }^{* *} A R B$, angiotensin II receptor blockers.

${ }_{\# \# D H P-C C B}$, dihydropyridine calcium channel blockers.

$p<0.0001)$ and had a level of hemoglobin A1c (HbAlc) of $5.9 \pm$ $0.5 \%$. All prediabetic patients were recommended for lifestyle modification as a measure for diabetic prevention except one patient received metformin at the dosage of 1,000 mg daily.

Serum pentosidine was slightly lower in the prediabetic group than that in the normoglycemic group $(3.0 \pm 1.8 \mathrm{ng} / \mathrm{ml}$ vs $4.1 \pm$ $2.2 \mathrm{ng} / \mathrm{ml}, p=0.071$ ); however, the difference did not reach statistical significance (Table 3). The level of serum pentosidine positively correlated with the level of $\mathrm{HbAlc}$ by univariate analysis $(r=0.614, p=0.044)$ in prediabetic population, suggesting that hyperglycemia had an impact on the accumulation of AGEs. Serum sRAGE, a decoy receptor of AGEs, and also the ratio between sRAGE and pentosidine (sRAGE-pentosidine ratio), were comparable in both prediabetic and normoglycemic groups (Table 3 ). In addition, serum interleukin-1 $\beta$ (IL-1 $\beta$ ) and tumor necrosis factor- $\alpha$ (TNF$\alpha$ ), markers of systemic inflammation, were comparable in both prediabetic and normoglycemic groups (Table 3). Therefore, individuals in both prediabetic and normoglycemic groups had comparable baseline characteristics (Tables 2, 3); FPG was the only parameter that showed significant difference between the two groups (Table 2).

\section{Preserved Osteogenic Differentiation, and Suppressed Expression of Both AGER and Apoptotic-Related Signals in PBMC Derived From Patients With Prediabetes}

The levels of expression of multiple osteoblast-specific genes, namely, ALPL, BGLAP, COL1A1, and RUNX2/PPAR were measured in the PBMC-isolated from both prediabetic and normoglycemic groups to define osteogenic differentiation. The PBMC derived from patients with prediabetes showed a preservation of osteogenic differentiation potential. The PBMCisolated from 25 patients with prediabetes (25/28) expressed ALPL, BGLAP, COL1A1, and RUNX2/PPAR and the PBMCisolated from 15 patients with normoglycemia (15/17) expressed those genes. Therefore, the PBMC derived from both prediabetic and normoglycemic groups had a comparable rate of osteogenic differentiation $(89.3$ and $88.2 \%, p=1.000)$. Among the PBMC showing differentiation toward osteoblast, the levels of osteoblast-specific gene expression were not significantly different between the normoglycemic and prediabetic groups, namely, ALPL ( $7.0 \pm 10.2$ vs $6.4 \pm 3.5, p=0.837)$, COL1A1 (9.30 \pm 9.2 vs $10.1 \pm 7.3, p=0.779), B G A L P(5.2 \pm 7.8$ vs $6.3 \pm 5.5, p=0.588)$, and RUNX2/PPAR (5.1 \pm 3.0 vs $8.0 \pm 7.6, p=0.239)$ (Figure 1).

TABLE 3 | Serum Pentosidine, sRAGE and Inflammation Markers in the Study Participants.

\begin{tabular}{lccc}
\hline Parameter & Normoglycemia $(\mathbf{n}=\mathbf{1 7})$ & Prediabetes $(\mathbf{n}=\mathbf{2 8})$ & $\boldsymbol{p}$-value \\
\hline Pentosidine $(\mathrm{ng} / \mathrm{ml})$ & $4.1 \pm 2.2$ & $3.0 \pm 1.8$ & 0.071 \\
Soluble RAGE $(\mathrm{sRAGE})(\mathrm{pg} / \mathrm{ml})$ & $610.4 \pm 352.3$ & $539.9 \pm 380.7$ & 0.539 \\
SRAGE-Pentosidine ratio $(\mathrm{pg} / \mathrm{hg})$ & $196.6 \pm 181.8$ & $245.6 \pm 232.4$ & 0.462 \\
Interleukin-1 $\beta$ (pg/ml) & $0.7 \pm 0.9$ & $0.7 \pm 0.7$ & 0.785 \\
Tumor necrosis factor- $\alpha(\mathrm{pg} / \mathrm{ml})$ & $1.7 \pm 3.6$ & $2.6 \pm 2.8$
\end{tabular}




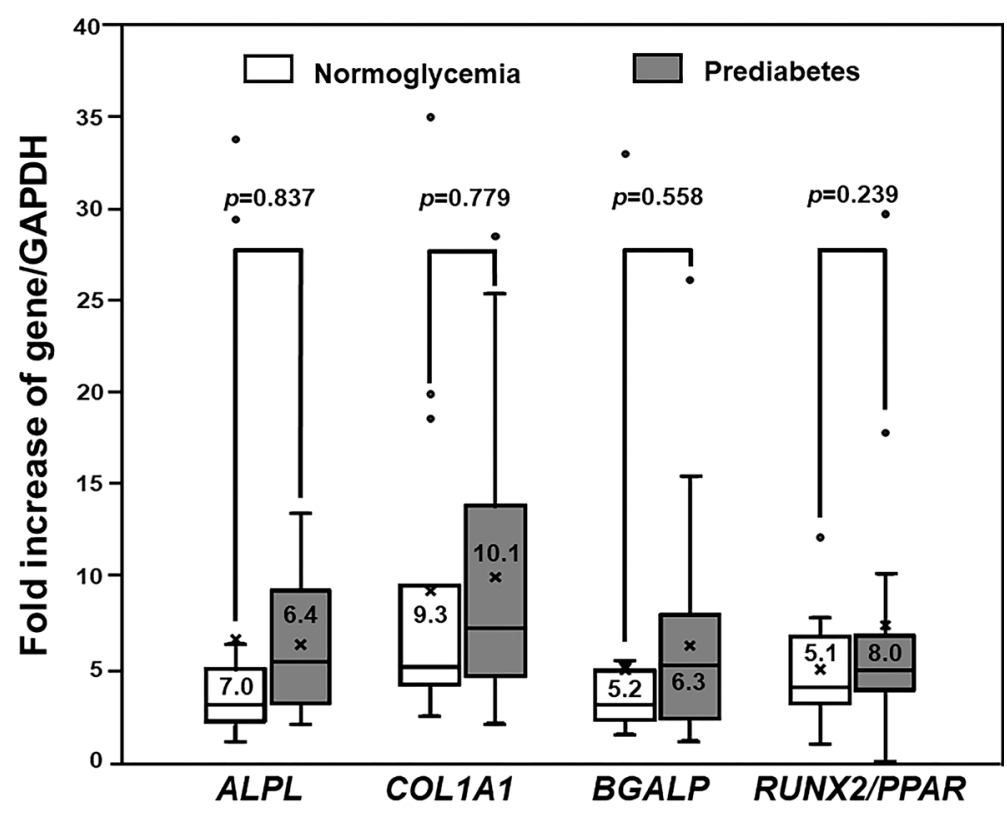

FIGURE 1 | The Expression of Osteogenic Differentiation Markers. Box and whisker plots to show a comparison of the expression of osteoblast-specific genes between participants with normoglycemia and patients with prediabetes (mean \pm SD). Both prediabetic and normoglycemic groups had comparable levels of osteoblast-specific gene expression, namely, ALPL, COL1A1, BGALP, and RUNX2/PPAR r ratio (RUNX2/PPAR).

According to the results of osteogenic differentiation in this study, we further performed backward power calculations to confirm that our sample size had adequate power to demonstrate a comparable differentiation ability between the prediabetic and normoglycemic groups (51). As a result, our study had $80 \%$ power to demonstrate non-inferior potential for osteogenic differentiation in the prediabetic group compared to the normoglycemic group at a margin of equivalence of $23 \%$.

Our previous studies demonstrated impaired osteogenic differentiation in PBMC isolated from patients with type 2 diabetes $(36,37)$, as well as cellular RAGE overexpression only in PBMC showing an impaired ability to differentiate toward osteoblast (37). Therefore, that cellular RAGE overexpression was conceivably associated with the impairment of osteogenic differentiation found in patients with type 2 diabetes. Since we demonstrated a preservation of osteogenic differentiation potential in PBMC derived from prediabetic patients, it was interesting to determine how cellular RAGE expression changes in those cells. To determine the alteration of cellular RAGE expression in patients with prediabetes showing preserved osteogenic differentiation, the level of AGER gene expression was compared between groups with prediabetes and normoglycemia. The PBMC showing poor osteogenic differentiation, two in the normoglycemic group and three in the prediabetic group, were excluded from the following analysis due to very small counts in these groups. Since pentosidine has been documented in other studies as an $A G E R$ enhancer $(28,29)$, and age was demonstrated in our previous study as a predictor of osteogenic differentiation potential (37), the level of $A G E R$ expression in prediabetic participants was compared with the level of AGER expression in age- and pentosidine-matched normoglycemic individuals. Interestingly, the AGER expression was significantly suppressed in individuals with prediabetes compared to age- and pentosidine-matched normoglycemic individuals $(0.5 \pm 0.6$ vs $1.0 \pm 0.0, p=0.003)$ (Figure 2). Since the activation of RAGE gives rise to the activation of its downstream apoptotic pathway, the levels of BAX and BCL2 expression were measured to determine whether the apoptoticassociated signals were also suppressed in prediabetic individuals. In concordant with $A G E R$ expression, $B A X / B C L 2$ expression ratio was significantly suppressed in prediabetic participants compared to those with normoglycemia $(0.7 \pm 0.5$ vs $1.0 \pm 0.0, p=0.010$ ) (Figure 2). Furthermore, the level of $A G E R$ expression significantly correlated with the level of the $B A X / B C L 2$ expression ratio $(r=0.986, p<0.0001)$. Therefore, the cellular RAGE suppression shown in prediabetes was conceivably a protective factor against cellular apoptosis and also osteogenic differentiation defects.

\section{Serum Pentosidine was an Independent Risk Factor for Determining AGER Expression in Individuals With Prediabetes}

Since the lower level of AGER expression was conceivably associated with the preservation of osteogenic differentiation of PBMC isolated from prediabetic individuals, the factors influencing that $A G E R$ suppression were valuable to be elucidated. Univariate analysis showed that AGER expression positively correlated with serum pentosidine $(r=0.513, p=$ $0.006)$, but inversely correlated with the duration of being prediabetic $(r=-0.480, p=0.011)$. Next, we performed the 


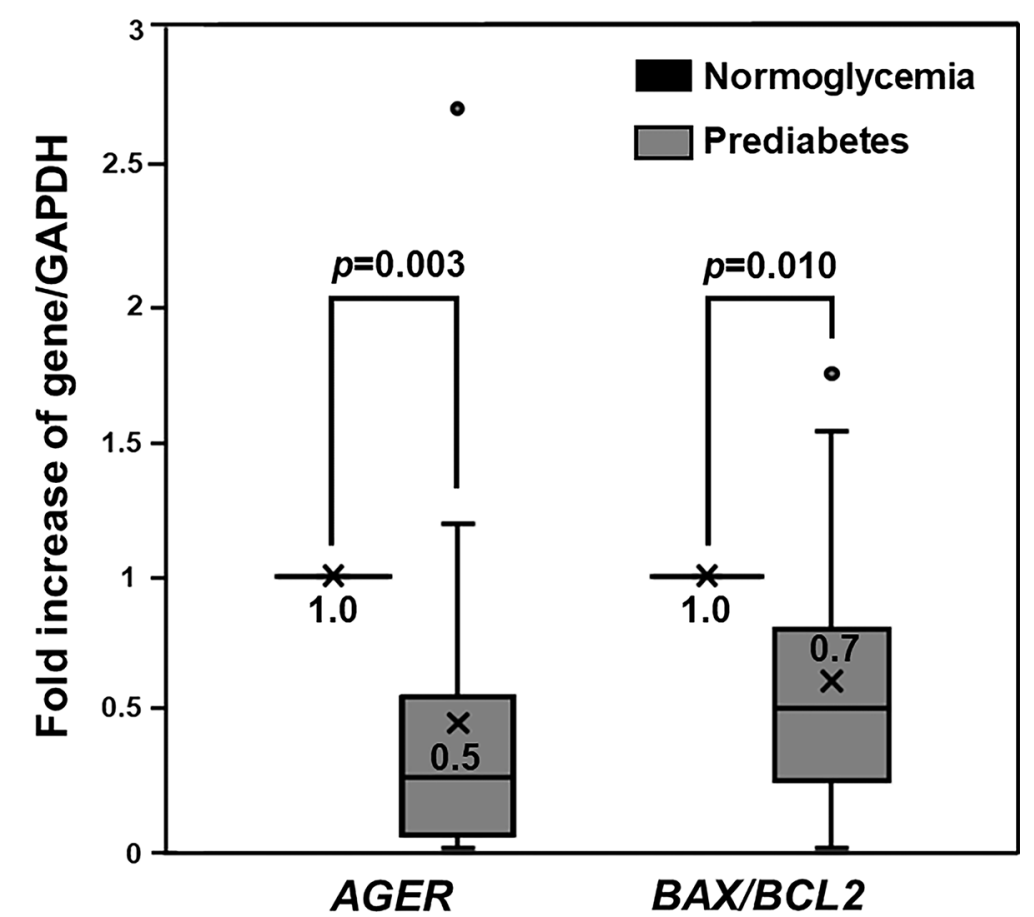

FIGURE 2 | The Expression of AGER, BAX, and BCL2 Genes. Box and whisker plots to show a comparison of AGER and BAX/BCL2 ratio between PBMC-isolated from individuals with normoglycemia and prediabetes. AGER and BAX/BCL2 ratio in PBMC-isolated from individuals with prediabetes were significantly suppressed by 2 and 1.4 folds, respectively, compared to those in age- and pentosidine-matched PBMC-isolated from participants with normoglycemia.

multivariate analysis using backward linear regression analysis to identify an independent risk factor for the expression of AGER. In addition to serum pentosidine and duration of being prediabetic, we included another 4 parameters that showed a correlation with either serum pentosidine or duration of being prediabetic in the further analysis because those factors may influence the expression of AGER. These 4 factors were as follows: HbAlc, which showed a positive correlation with serum pentosidine in the prediabetic group $(r=0.614, p=$ $0.044)$; and FPG $(r=0.425, p=0.007)$, FRAX-H $(r=0.477$, $p=0.004)$, and FRAX-O $(r=0.49, p=0.003)$, which showed a positive correlation with the duration of being prediabetic. Upon performing the backward linear regression analysis, the multivariate analysis showed that only serum pentosidine significantly associated with the expression of AGER ( $r=$ 0.879, $p=0.002$ ), indicating that serum pentosidine was an independent risk factor for determining AGER expression. Therefore, in this present study, serum pentosidine was the only factor identified as independently contributing to the expression of AGER.

We next determined the potential of using serum pentosidine as a predictor of $A G E R$ suppression in prediabetic individuals. We categorized the AGER expression into two groups, including the AGER suppression group as defined by an AGER expression level of less than 1 and the AGER non-suppression group as defined by the level of $A G E R$ expression as equal to 1 or higher. Regarding serum pentosidine, the cut-off level of serum pentosidine to predict the level of AGER expression with the highest sensitivity and specificity was calculated. As a result, the cut-off level of serum pentosidine to predict the level of AGER expression with $90.9 \%$ sensitivity and $46.7 \%$ specificity was 2.1 $\mathrm{ng} / \mathrm{ml}$. Therefore, we applied this value of serum pentosidine for further analysis. After categorizing patients into two groups according to the level of AGER expression, we next performed logistic regression analysis to determine whether the proposed cut-off point of serum pentosidine predicted the occurrence of AGER suppression. When the AGER expression was categorized into two groups, serum pentosidine at the cut-off level of $2.1 \mathrm{ng} /$ $\mathrm{ml}(\mathrm{OR} 8.75$; 95\% CI 0.88-86.6, $p=0.064)$ and FPG at the cut-off level of $100 \mathrm{mg} / \mathrm{dl}$ (OR 0.15; 95\% CI 0.02-0.94, $p=0.043$ ) showed an association with the group of $A G E R$ expression. As demonstrated in Figure 3, the area under the ROC curve (AUC) of serum pentosidine to predict the AGER expression was 0.688 . In addition to serum pentosidine, when we put the cut-off point of FPG at the prediabetic range into the model, the AUC significantly increased from 0.688 to 0.803 ( 0.803 vs 0.688 , $p=0.048$ ) (Figure 3). This improvement in AUC indicated that serum pentosidine was a good predictor for AGER expression in prediabetic patients. The predictive probability of $A G E R$ suppression by serum pentosidine at the cut-off point of less than $2.1 \mathrm{ng} / \mathrm{ml}$ and FPG at the cut-off point of prediabetes was 0.97 , which means that $97 \%$ of patients with prediabetes were correctly classified into the AGER suppression group if those patients had a serum pentosidine level lower than $2.1 \mathrm{ng} / \mathrm{ml}$.

The results of linear regression analysis indicated that serum pentosidine was an independent risk factor for AGER expression. 


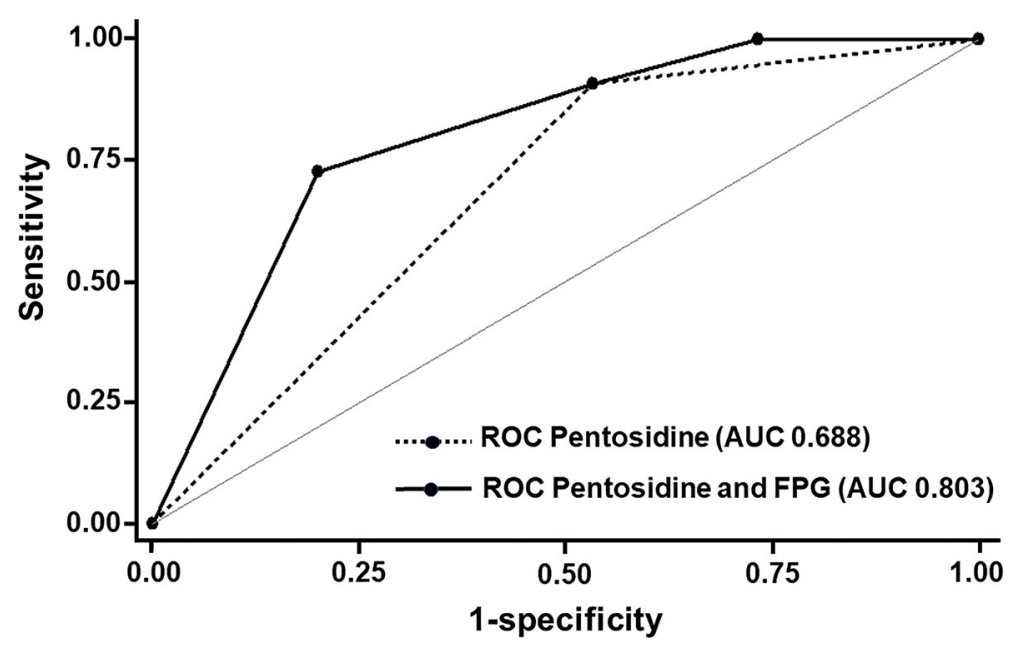

FIGURE 3 | ROC and Area under ROC (AUC) for the Prediction of the expression of AGER. With serum pentosidine at the cut-off level of $2.1 \mathrm{ng} / \mathrm{ml}$, the AUC of serum pentosidine to predict the expression of AGER was 0.688 . With the combination of both serum pentosidine at the cut-off level of $2.1 \mathrm{ng} / \mathrm{ml}$ and fasting plasma glucose at the cut-off level of $100 \mathrm{mg} / \mathrm{dl}$ into the model, the AUC significantly increased from 0.688 to $0.803(0.803 \mathrm{vs} 0.688, p=0.048)$.

However, from the result of logistic regression analysis, serum pentosidine was shown as a good predictor for AGER expression only in the prediabetic group. Therefore, our study demonstrated the potential of using serum pentosidine as a predictor of $A G E R$ expression in prediabetic individuals. With a serum pentosidine level of lower than $2.1 \mathrm{ng} / \mathrm{ml}$, there was an extremely high possibility of the expression of AGER being suppressed in prediabetic individuals. Since the AGER suppression shown in the prediabetic group was conceivably a protective factor against cellular apoptosis as well as osteogenic differentiation defects, it may be inferred that a serum pentosidine level of lower than 2.1 $\mathrm{ng} / \mathrm{ml}$ is a protective factor against cellular apoptosis and impaired osteogenic differentiation in this prediabetic group.

\section{DISCUSSION}

This study demonstrated the preservation of osteogenic differentiation ability in the PBMC derived from prediabetic individuals as defined by the impaired fasting glucose criteria. In addition, the suppression of AGER and apoptotic-related signals was demonstrated in those prediabetic-derived PBMC that showed the preservation of osteogenic differentiation potential. Serum pentosidine was shown as an independent risk factor for the cellular RAGE expression. In addition, the level of serum pentosidine of lower than $2.1 \mathrm{mg} / \mathrm{dl}$ was demonstrated as being a good predictor for the occurrence of $A G E R$ suppression in individuals with prediabetes.

In high fat diet-induced obesity and prediabetic animal models, a decrease in osteoblast proliferation, differentiation, and survival, and also a deterioration of bone microarchitecture have been consistently demonstrated $(41,42)$. However, in humans, it has been shown that individuals with prediabetes retain a preservation of both bone mineral density (17-20) and bone material strength (24). Moreover, there have been inconsistent reports involving the increment of hip fracture in prediabetic population $(19,21-23)$. Therefore, the detrimental effects of prediabetes on the skeleton remain to be elucidated in prediabetic patients. This study involving osteogenic differentiation ability of the stem cells in prediabetic patients may give rise to additional information on this obscure issue. Furthermore, it is valuable to elucidate whether there is an osteogenic differentiation defect at the prediabetic stage as a measure for establishing a skeletal health strategy in type 2 diabetes in the future. In this study, we demonstrated the preservation of osteogenic differentiation in PBMC isolated from patients with prediabetes. The PBMC derived from this prediabetic group showed the same rate of differentiation toward osteoblasts as the rate in the normoglycemic group. In addition, they also had the same degree of osteoblast-specific gene expression as that in normoglycemic individuals. Therefore, being prediabetic as diagnosed by IFG, did not show a detrimental effect on the osteogenic differentiation potential of the PBMC, which is probably linked to the preservation of bone mineral density and bone microarchitecture which has been shown in several previous studies and may favor a neutral fracture risk in prediabetic individuals.

Our previous study, performed in patients with type 2 diabetes, demonstrated AGER overexpression only in PBMC showing poor osteogenic differentiation ability but not in the PBMC showing preserved osteogenic differentiation (37), suggesting a linkage between AGER overexpression and a defect in osteogenic differentiation in PBMC derived from individuals with type 2 diabetes. In this study, we demonstrated the preservation of osteogenic differentiation in the PBMC isolated from patients with prediabetes. Therefore, it is interesting to see how cellular RAGE expression alteration in this group of patients with preserved osteogenic differentiation. 
In this study, when we performed analysis of cellular RAGE expression in $\mathrm{PBMC}$ showing preserved osteogenic differentiation, we demonstrated a lower level of $A G E R$ expression in the PBMC derived from the prediabetic group compared to those from the normoglycemic group, indicating cellular RAGE suppression in prediabetes with preserved osteogenic differentiation ability. Therefore, it is noteworthy to state that the level of cellular RAGE expression would influence osteogenic differentiation in both prediabetic and diabetic individuals, ranging from suppression in prediabetes to overexpression in type 2 diabetes. Our results regarding AGER suppression in prediabetes were consistent with a previous report (52). Ruelas and colleagues (52) demonstrated lower expression levels of both the AGER gene and RAGE protein in PBMC derived from patients with prediabetes and insulin resistance than those in the PBMC-isolated from normal healthy volunteers. However, they did not determine the differentiation potential of those isolated PBMC (52). Even though AGER gene suppression was repetitively shown in both our and previous studies, the underlying mechanism leading to the suppression of $A G E R$ in prediabetes remains to be elucidated. To date, several signal alterations have been shown to maintain normal bone metabolism in prediabetes. In an animal model, Mohammad and colleagues (53) reported a ligand-induced downregulation of TLR4 in bone marrow-derived macrophages isolated from prediabetic mice while TLR4 was constantly high in diabetic mice. Zhang and colleagues (54) demonstrated that the decrement of TLR4 reduced hyperglycemia-induced osteoblast apoptosis, promoted bone mineralization, and improved bone structure in a rat model. Therefore, the downregulation of TLR4 occurred at a prediabetic stage to maintain osteoblast survival and bone integrity. In contrast, Bhansali and colleagues (55) demonstrated an increase in mitophagy-related markers and mitochondrial mass in PBMC derived from patients with prediabetes but showed a decrease in those mitophagy-related markers and mitochondrial mass in cases with type 2 diabetes. Later, Gao and colleagues (56) showed that the regulation of mitochondrial stress by the prevention of oxidative damage to the mitochondria is required during osteogenic differentiation and bone formation. In addition, Dobson and colleagues (57) demonstrated that mitochondrial dysfunction entailed impaired osteogenesis and accelerated age-related osteoporosis. Therefore, the upregulation of mitophagy which occurred at the prediabetic stage was essential to maintain normal osteoblast development and function. Following previous reports on changes in signal regulation in prediabetes, we hypothesized that the downregulation of AGEs-dependent RAGE activation occurred at the prediabetic stage. The downregulation of AGEs-dependent RAGE activation would yield a lower degree of RAGE stimulation even in the presence of the same amount of AGEs, as demonstrated by the significant suppression of $A G E R$ in prediabetic individuals compared to age- and pentosidinematched normoglycemic participants in our study. In addition, the downregulation of AGEs-dependent RAGE activation may lead to a lower rate of AGEs formation even in the presence of mild hyperglycemia, as demonstrated by the insignificant lower serum pentosidine level in the prediabetic group compared to the normoglycemic group in our study. Even though the underlying mechanism for the downregulation of AGEs-dependent RAGE activation remains to be further elucidated, it is noteworthy to state that the downregulation of AGEs-dependent RAGE activation that occurred at the prediabetic stage is conceivably one of the mechanisms to promote osteoblast differentiation and survival, which then maintains osteogenesis and bone integrity in individuals with prediabetes.

Since the suppression of $A G E R$ conceivably contributes to a decrease in cellular apoptosis and preservation of osteogenic differentiation in prediabetes, the suppression of $A G E R$ would be a good predictive factor for the maintenance of skeletal integrity in prediabetes. However, the measurement of AGER expression in the PBMC required multiple steps that were mostly performed on research scales. Further analysis was conducted to determine the independent risk factors of AGER expression. If this independent risk factor could be analyzed easily, it may be used as a surrogate marker for cellular RAGE expression and the differentiation ability of the PBMC. Using linear regression analysis, serum pentosidine was shown to be an independent risk factor for AGER expression. Since the cut-off point of serum pentosidine level to predict the adverse outcomes of AGEs has never been reported, the cut-off point of serum pentosidine was calculated for the highest sensitivity and specificity in our study. A serum pentosidine level of $2.1 \mathrm{ng} / \mathrm{ml}$ showed the highest sensitivity and specificity to predict the expression of AGER. Logistic regression analysis was then performed to determine the predictive potential of this proposed cut-off point. Our results showed that this proposed cut-off point was a good predictor for $A G E R$ expression only in the prediabetic group (AUC 0.803). Since serum pentosidine is much easier to measure than cellular RAGE expression, it is more applicable, especially in the clinic, to use serum pentosidine as a surrogate marker for cellular RAGE expression and differentiation potential of the PBMC. Serum pentosidine has previously been shown to be useful as a predictor of chronic microvascular $(2,4)$ and macrovascular complications (3), and also vertebral fractures $(14,39,40)$ in a diabetic population. A higher level of serum pentosidine would increase the risk of diabetic complications and fracture; however, a cut-off point for clinical prediction has never been proposed. Our study provided another aspect of using serum pentosidine as a protective predictive factor in prediabetes. A level of serum pentosidine lower than the threshold of $2.1 \mathrm{ng} / \mathrm{ml}$ was found to be associated with lower AGER expression level and may relate to the preservation of osteogenic differentiation in prediabetes.

This study is the first study to demonstrate the preservation of osteogenic differentiation in PBMC derived from prediabetic individuals, and being the first to show the suppression of cellular RAGE and apoptotic-related signals in those PBMC with a preserved osteogenic differentiation ability. In addition, this study proposes the possibility of using serum pentosidine as a surrogate marker for cellular RAGE expression and osteogenic differentiation ability in prediabetic patients. However, this evidence should be interpreted with caution as there are several limitations. First, this study only demonstrated signal activation by mRNA levels due to 
the limited number of isolated cells from the relatively small 35-40 $\mathrm{ml}$ sample of peripheral blood collected from recruited patients. Due to the possibility of the gene being transcripted but not translated into proteins, the AGER suppression probably does not lead to lower RAGE activation. Second, this study was a small study, including 28 individuals with prediabetes and 17 participants with normoglycemia, which may have impacted the power of the study. This study was shown to have $80 \%$ power to demonstrate non-inferior potential for osteogenic differentiation in the prediabetic group compared to the normoglycemic group at a margin of equivalence of $23 \%$, so the small difference in osteogenic differentiation potential of less than $23 \%$ would not be detected by this study. Third, this study demonstrated only the pattern of association between parameters, so the causes and effects of those parameters cannot be concluded. Regarding the association between AGER suppression and osteoblast differentiation, studies involving RAGE overexpression would clarify whether cellular RAGE suppression directly yields to preserved osteoblast differentiation. Fourth, the cut-off level of serum pentosidine may not be universally equal to $2.1 \mathrm{ng} / \mathrm{dl}$. This proposed threshold may need to be verified before diagnostic application in the clinic. Even though the ELISA test kit used for the measurement of serum pentosidine in this study has a wide range of detection (0.47-50 ng/ dl) and has a coefficient of variation of less than $10 \%$ according to the manufacturer information, this test kit is established for a research scale but not for diagnostic purposes. Last, this study was a cross-sectional study which had several unexpected confounding factors caused by the nature of this type of study. Even though all baseline characteristics of the enrolled participants were generally comparable, those unexpected confounding factors might influence the results of the study.

\section{DATA AVAILABILITY STATEMENT}

The original contributions presented in the study are included in the article/supplementary material. Further inquiries can be directed to the corresponding author.

\section{REFERENCES}

1. American Diabetes Associtaion. 2. Classification and Diagnosis of Diabetes: Standards of Medical Care in Diabetes-2021. Diabetes Care (2021) 44 (Suppl 1):S15-33. doi: 10.2337/dc21-S002

2. Garay-Sevilla ME, Regalado JC, Malacara JM, Nava LE, Wróbel-Zasada K, Castro-Rivas A, et al. Advanced Glycosylation End Products in Skin, Serum, Saliva and Urine and its Association With Complications of Patients With Type 2 Diabetes Mellitus. J Endocrinol Invest (2005) 28(3):223-30. doi: $10.1007 /$ bf03345377

3. Kilhovd BK, Juutilainen A, Lehto S, Rönnemaa T, Torjesen PA, Hanssen KF, et al. Increased Serum Levels of Advanced Glycation Endproducts Predict Total, Cardiovascular and Coronary Mortality in Women With Type 2 Diabetes: A Population-Based 18 Year Follow-Up Study. Diabetologia (2007) 50(7):1409-17. doi: 10.1007/s00125-007-0687-Z

4. Ng ZX, Chua KH, Iqbal T, Kuppusamy UR. Soluble Receptor for Advanced Glycation End-Product (sRAGE)/Pentosidine Ratio: A Potential Risk Factor Determinant for Type 2 Diabetic Retinopathy. Int J Mol Sci (2013) 14 (4):7480-91. doi: 10.3390/ijms14047480

\section{ETHICS STATEMENT}

The studies involving human participants were reviewed and approved by the Research Ethics Committee of the Faculty of Medicine, Chiang Mai University. The patients/participants provided their written informed consent to participate in this study.

\section{AUTHOR CONTRIBUTIONS}

MP was involved in conceptualization, funding acquisition, methodology, formal analysis, original draft writing, reviewing and editing of the manuscript. PP was involved in the methodology, original draft writing, reviewing and editing of the manuscript. NC was involved in funding acquisition, and reviewing and editing of the manuscript. PK was involved in the reviewing and editing of the manuscript. All authors listed have made a substantial, direct, and intellectual contribution to the work and approved it for publication.

\section{FUNDING}

This work is supported by the Thailand Research Fund MRG5480270 (MP), Merck (MP), the NSTDA Research Chair Grant from the National Science and Technology Development Agency (NC), and the Chiang Mai University Center of Excellence Award (NC). All funders had no role in study design, data collection and analysis, preparation of the manuscript, or decision to publish.

\section{ACKNOWLEDGMENTS}

The authors would like to thank Mrs. Antika Wongtanee for performing the statistical analysis.

5. Yamanaka M, Matsumura T, Ohno R, Fujiwara Y, Shinagawa M, Sugawa H, et al. Non-Invasive Measurement of Skin Autofluorescence to Evaluate Diabetic Complications. J Clin Biochem Nutr (2016) 58(2):135-40. doi: $10.3164 /$ jcbn.15-132

6. Ali MK, Bullard KM, Saydah S, Imperatore G, Gregg EW. Cardiovascular and Renal Burdens of Prediabetes in the USA: Analysis of Data From Serial CrossSectional Surveys, 1988-2014. Lancet Diabetes Endocrinol (2018) 6(5):392403. doi: 10.1016/s2213-8587(18)30027-5

7. Gujral UP, Jagannathan R, He S, Huang M, Staimez LR, Wei J, et al. Association Between Varying Cut-Points of Intermediate Hyperglycemia and Risk of Mortality, Cardiovascular Events and Chronic Kidney Disease: A Systematic Review and Meta-Analysis. BMJ Open Diabetes Res Care (2021) 9(1):e001776. doi: 10.1136/bmjdrc-2020-001776

8. Gateva AT, Assyov YS, Tsakova AD, Kamenov ZA. Serum AGEs and sRAGE Levels are Not Related to Vascular Complications in Patients With Prediabetes. Diabetes Metab Syndr (2019) 13(2):1005-10. doi: 10.1016/j.dsx.2019.01.014

9. Shu A, Yin MT, Stein E, Cremers S, Dworakowski E, Ives R, et al. Bone Structure and Turnover in Type 2 Diabetes Mellitus. Osteoporos Int (2012) 23 (2):635-41. doi: 10.1007/s00198-011-1595-0 
10. Reyes-García R, Rozas-Moreno P, López-Gallardo G, García-Martín A, Varsavsky M, Avilés-Perez MD, et al. Serum Levels of Bone Resorption Markers are Decreased in Patients With Type 2 Diabetes. Acta Diabetol (2013) 50(1):47-52. doi: 10.1007/s00592-011-0347-0

11. Paccou J, Ward KA, Jameson KA, Dennison EM, Cooper C, Edwards MH. Bone Microarchitecture in Men and Women With Diabetes: The Importance of Cortical Porosity. Calcif Tissue Int (2016) 98(5):465-73. doi: 10.1007/ s00223-015-0100-8

12. Janghorbani M, Van Dam RM, Willett WC, Hu FAB. Systematic Review of Type 1 and Type 2 Diabetes Mellitus and Risk of Fracture. Am J Epidemiol (2007) 166(5):495-505. doi: 10.1093/aje/kwm106

13. Vestergaard P. Discrepancies in Bone Mineral Density and Fracture Risk in Patients With Type 1 and Type 2 Diabetes-A Meta-Analysis. Osteoporos Int (2007) 18(4):427-44. doi: 10.1007/s00198-006-0253-4

14. Yamamoto M, Yamaguchi T, Yamauchi M, Kaji H, Sugimoto T. Bone Mineral Density Is Not Sensitive Enough to Assess the Risk of Vertebral Fractures in Type 2 Diabetic Women. Calcif Tissue Int (2007) 80(6):353-8. doi: 10.1007/ s00223-007-9003-7

15. Ma L, Oei L, Jiang L, Estrada K, Chen H, Wang Z, et al. Association Between Bone Mineral Density and Type 2 Diabetes Mellitus: A Meta-Analysis of Observational Studies. Eur J Epidemiol (2012) 27(5):319-32. doi: 10.1007/ s10654-012-9674-x

16. Jiajue R, Jiang Y, Wang O, Li M, Xing X, Cui L, et al. Suppressed Bone Turnover was Associated With Increased Osteoporotic Fracture Risks in NonObese Postmenopausal Chinese Women With Type 2 Diabetes Mellitus. Osteoporos Int (2014) 25(8):1999-2005. doi: 10.1007/s00198-014-2714-5

17. Mitchell A, Fall T, Melhus H, Wolk A, Michaëlsson K, Byberg L. Type 2 Diabetes in Relation to Hip Bone Density, Area, and Bone Turnover in Swedish Men and Women: A Cross-Sectional Study. Calcif Tissue Int (2018) 103(5):501-11. doi: 10.1007/s00223-018-0446-9

18. Holloway-Kew KL, Marijanovic N, De Abreu LLF, Sajjad MA, Pasco JA, Kotowicz MA. Bone Mineral Density in Diabetes and Impaired Fasting Glucose. Osteoporos Int (2019) 30(9):1799-806. doi: 10.1007/s00198-01905108-1

19. Chen C, Chen Q, Nie B, Zhang H, Zhai H, Zhao L, et al. Trends in Bone Mineral Density, Osteoporosis, and Osteopenia Among U.S. Adults With Prediabetes, 2005-2014. Diabetes Care (2020) 43(5):1008-15. doi: 10.2337/ dc19-1807

20. Liu W, Wang C, Hao J, Yin L, Wang Y, Li W. Association Between Metabolic Syndrome and Osteoporosis: A Systematic Review and Meta-Analysis. Int J Endocrinol (2021) 2021:6691487. doi: 10.1155/2021/6691487

21. Park HY, Han K, Kim Y, Kim YH, Sur YJ. The Risk of Hip Fractures in Individuals Over 50 Years Old With Prediabetes and Type 2 Diabetes - A Longitudinal Nationwide Population-Based Study. Bone (2021) 142:115691. doi: 10.1016/j.bone.2020.115691

22. Dominic E, Brozek W, Peter RS, Fromm E, Ulmer H, Rapp K, et al. Metabolic Factors and Hip Fracture Risk in a Large Austrian Cohort Study. Bone Rep (2020) 12:100244. doi: 10.1016/j.bonr.2020.100244

23. Iki M, Fujita Y, Kouda K, Yura A, Tachiki T, Tamaki J, et al. Hyperglycemic Status is Associated With an Elevated Risk of Osteoporotic Fracture in Community-Dwelling Elderly Japanese Men: The Fujiwara-Kyo Osteoporosis Risk in Men (FORMEN) Cohort Study. Bone (2019) 121:1006. doi: 10.1016/j.bone.2019.01.005

24. Dawson-Hughes B, Bouxsein M, Shea K. Bone Material Strength in Normoglycemic and Hyperglycemic Black and White Older Adults. Osteoporos Int (2019) 30(12):2429-35. doi: 10.1007/s00198-019-05140-1

25. Xie J, Méndez JD, Méndez-Valenzuela V, Aguilar-Hernández MM. Cellular Signalling of the Receptor for Advanced Glycation End Products (RAGE). Cell Signal (2013) 25(11):2185-97. doi: 10.1016/j.cellsig.2013.06.013

26. Ng ZX, Kuppusamy UR, Iqbal T, Chua KH. Receptor for Advanced Glycation End-Product (RAGE) Gene Polymorphism 2245G/A is Associated With ProInflammatory, Oxidative-Glycation Markers and sRAGE in Diabetic Retinopathy. Gene (2013) 521(2):227-33. doi: 10.1016/j.gene.2013.03.062

27. Alikhani M, Alikhani Z, Boyd C, MacLellan CM, Raptis M, Liu R, et al. Advanced Glycation End Products Stimulate Osteoblast Apoptosis via the MAP Kinase and Cytosolic Apoptotic Pathways. Bone (2007) 40(2):345-53. doi: 10.1016/j.bone.2006.09.011
28. Franke S, Siggelkow H, Wolf G, Hein G. Advanced Glycation Endproducts Influence the mRNA Expression of RAGE, RANKL and Various Osteoblastic Genes in Human Osteoblasts. Arch Physiol Biochem (2007) 113(3):154-61. doi: 10.1080/13813450701602523

29. Mercer N, Ahmed H, Etcheverry SB, Vasta GR, Cortizo AM. Regulation of Advanced Glycation End Product (AGE) Receptors and Apoptosis by AGEs in Osteoblast-Like Cells. Mol Cell Biochem (2007) 306(1-2):87-94. doi: 10.1007/s11010-007-9557-8

30. Okazaki K, Yamaguchi T, Tanaka K, Notsu M, Ogawa N, Yano S, et al. Advanced Glycation End Products (AGEs), But Not High Glucose, Inhibit the Osteoblastic Differentiation of Mouse Stromal ST2 Cells Through the Suppression of Osterix Expression, and Inhibit Cell Growth and Increasing Cell Apoptosis. Calcif Tissue Int (2012) 91(4):286-96. doi: 10.1007/s00223012-9641-2

31. Liu J, Mao J, Jiang Y, Xia L, Mao L, Wu Y, et al. AGEs Induce Apoptosis in Rat Osteoblast Cells by Activating the Caspase-3 Signaling Pathway Under a High-Glucose Environment In Vitro. Appl Biochem Biotechnol (2016) 178 (5):1015-27. doi: 10.1007/s12010-015-1925-3

32. Chen H, Liu W, Wu X, Gou M, Shen J, Wang H. Advanced Glycation End Products Induced IL-6 and VEGF-A Production and Apoptosis in OsteocyteLike MLO-Y4 Cells by Activating RAGE and ERK1/2, P38 and STAT3 Signalling Pathways. Int Immunopharmacol (2017) 52:143-9. doi: 10.1016/ j.intimp.2017.09.004

33. Tang SY, Zeenath U, Vashishth D. Effects of Non-Enzymatic Glycation on Cancellous Bone Fragility. Bone (2007) 40(4):1144-51. doi: 10.1016/ j.bone.2006.12.056

34. Poundarik AA, Wu PC, Evis Z, Sroga GE, Ural A, Rubin M, et al. A Direct Role of Collagen Glycation in Bone Fracture. J Mech Behav BioMed Mater (2015) 52:120-30. doi: 10.1016/j.jmbbm.2015.08.012

35. Epstein S, Defeudis G, Manfrini S, Napoli N, Pozzilli P. Diabetes and Disordered Bone Metabolism (Diabetic Osteodystrophy): Time for Recognition. Osteoporos Int (2016) 27(6):1931-51. doi: 10.1007/s00198-0153454-x

36. Phimphilai $\mathrm{M}$, Pothacharoen $\mathrm{P}$, Kongtawelert $\mathrm{P}$, Chattipakorn N. Impaired Osteogenic Differentiation and Enhanced Cellular Receptor of Advanced Glycation End Products Sensitivity in Patients With Type 2 Diabetes. J Bone Mineral Metab (2017) 35(6):631-41. doi: 10.1007/s00774-016-0800-9

37. Phimphilai M, Pothacharoen P, Kongtawelert P. Age-Influenced Receptors of Advanced Glycation End Product Overexpression Associated With Osteogenic Differentiation Impairment in Patients With Type 2 Diabetes. Front Endocrinol (Lausanne) (2021) 12:726182. doi: 10.3389/fendo.2021. 726182

38. Yamamoto M, Yamaguchi T, Yamauchi M, Yano S, Sugimoto T. Serum Pentosidine Levels are Positively Associated With the Presence of Vertebral Fractures in Postmenopausal Women With Type 2 Diabetes. J Clin Endocrinol Metab (2008) 93(3):1013-9. doi: 10.1210/jc.2007-1270

39. Schwartz AV, Garnero P, Hillier TA, Sellmeyer DE, Strotmeyer ES, Feingold KR, et al. Pentosidine and Increased Fracture Risk in Older Adults With Type 2 Diabetes. J Clin Endocrinol Metab (2009) 94(7):2380-6. doi: 10.1210/ jc. $2008-2498$

40. Tamaki J, Kouda K, Fujita Y, Iki M, Yura A, Miura M, et al. Ratio of Endogenous Secretory Receptor for Advanced Glycation End Products to Pentosidine Predicts Fractures in Men. J Clin Endocrinol Metab (2018) 103 (1):85-94. doi: 10.1210/jc.2017-00929

41. Pramojanee SN, Phimphilai M, Kumphune S, Chattipakorn N, Chattipakorn SC. Decreased Jaw Bone Density and Osteoblastic Insulin Signaling in a Model of Obesity. J Dent Res (2013) 92(6):560-5. doi: 10.1177/0022034513485600

42. Ross DS, Yeh TH, King S, Mathers J, Rybchyn MS, Neist E, et al. Distinct Effects of a High Fat Diet on Bone in Skeletally Mature and Developing Male C57BL/6J Mice. Nutrients (2021) 13(5):1666. doi: 10.3390/nu13051666

43. Hass R, Kasper C, Böhm S, Jacobs R. Different Populations and Sources of Human Mesenchymal Stem Cells (MSC): A Comparison of Adult and Neonatal Tissue-Derived MSC. Cell Commun Signal (2011) 9:12. doi: 10.1186/1478-811x-9-12

44. Cesselli D, Beltrami AP, Rigo S, Bergamin N, D’Aurizio F, Verardo R, et al. Multipotent Progenitor Cells Are Present in Human Peripheral Blood. Circ Res (2009) 104(10):1225-34. doi: 10.1161/circresaha.109.195859 
45. Yang HS, Kim GH, La WG, Bhang SH, Lee TJ, Lee JH, et al. Enhancement of Human Peripheral Blood Mononuclear Cell Transplantation-Mediated Bone Formation. Cell Transplant (2011) 20(9):1445-52. doi: 10.3727/ 096368910x557272

46. Chong PP, Selvaratnam L, Abbas AA, Kamarul T. Human Peripheral Blood Derived Mesenchymal Stem Cells Demonstrate Similar Characteristics and Chondrogenic Differentiation Potential to Bone Marrow Derived Mesenchymal Stem Cells. J Orthop Res (2012) 30(4):634-42. doi: 10.1002/ jor. 21556

47. Valenti MT, Dalle Carbonare L, Donatelli L, Bertoldo F, Zanatta M, Lo Cascio V. Gene Expression Analysis in Osteoblastic Differentiation From Peripheral Blood Mesenchymal Stem Cells. Bone (2008) 43(6):1084-92. doi: 10.1016/ j.bone.2008.07.252

48. Centre for Metabolic Bone Diseases. The Fracture Risk Assessment Tool $\left(F R A X^{\circledR}\right)$ [Internet]. Sheffield: University of Sheffield; [Cited 2021 August 23]. Available at: https://www.sheffield.ac.uk/FRAX/tool.aspx?country=57.

49. Lian JB, Stein GS. Development of the Osteoblast Phenotype: Molecular Mechanisms Mediating Osteoblast Growth and Differentiation. Iowa Orthop J (1995) 15:118-40.

50. Czekanska EM, Stoddart MJ, Ralphs JR, Richards RG, Hayes JS. A Phenotypic Comparison of Osteoblast Cell Lines Versus Human Primary Osteoblasts for Biomaterials Testing. J BioMed Mater Res A (2014) 102(8):2636-43. doi: 10.1002/jbm.a.34937

51. Chow S, Wang H, Shao J. Large Sample Tests for Proportions. In: S Chow, J Shao, H Wang, editors. Sample Size Calculations in Clinical Research, 2nd ed. Boca Raton: Chapman \& Hall/CRC (2008). p. 83-115.

52. Ruelas Cinco EDC, Ruíz Madrigal B, Domínguez Rosales JA, Maldonado González M, de la Cruz Color L, Ramírez Meza SM, et al. Expression of the Receptor of Advanced Glycation End-Products (RAGE) and Membranal Location in Peripheral Blood Mononuclear Cells (PBMC) in Obesity and Insulin Resistance. Iran J Basic Med Sci (2019) 22(6):623-30. doi: 10.22038/ ijbms.2019.34571.8206

53. Mohammad MK, Morran M, Slotterbeck B, Leaman DW, Sun Y, Grafenstein $\mathrm{H}$, et al. Dysregulated Toll-Like Receptor Expression and Signaling in Bone Marrow-Derived Macrophages at the Onset of Diabetes in the Non-Obese
Diabetic Mouse. Int Immunol (2006) 18(7):1101-13. doi: 10.1093/intimm/ dxl045

54. Zhang Y, Shen X, Cheng L, Chen R, Zhao F, Zhong S, et al. Toll-Like Receptor 4 Knockout Protects Against Diabetic-Induced Imbalance of Bone Metabolism via Autophagic Suppression. Mol Immunol (2020) 117:12-9. doi: 10.1016/j.molimm.2019.10.025

55. Bhansali S, Bhansali A, Walia R, Saikia UN, Dhawan V. Alterations in Mitochondrial Oxidative Stress and Mitophagy in Subjects With Prediabetes and Type 2 Diabetes Mellitus. Front Endocrinol (Lausanne) (2017) 8:347. doi: 10.3389/fendo.2017.00347

56. Gao J, Feng Z, Wang X, Zeng M, Liu J, Han S, et al. SIRT3/SOD2 Maintains Osteoblast Differentiation and Bone Formation by Regulating Mitochondrial Stress. Cell Death Differ (2018) 25(2):229-40. doi: 10.1038/cdd.2017.144

57. Dobson PF, Dennis EP, Hipps D, Reeve A, Laude A, Bradshaw C, et al. Mitochondrial Dysfunction Impairs Osteogenesis, Increases Osteoclast Activity, and Accelerates Age Related Bone Loss. Sci Rep (2020) 10 (1):11643. doi: 10.1038/s41598-020-68566-2

Conflict of Interest: The authors declare that the research was conducted in the absence of any commercial or financial relationships that could be construed as a potential conflict of interest.

Publisher's Note: All claims expressed in this article are solely those of the authors and do not necessarily represent those of their affiliated organizations, or those of the publisher, the editors and the reviewers. Any product that may be evaluated in this article, or claim that may be made by its manufacturer, is not guaranteed or endorsed by the publisher.

Copyright ( 12022 Phimphilai, Pothacharoen, Chattipakorn and Kongtawelert. This is an open-access article distributed under the terms of the Creative Commons Attribution License (CC BY). The use, distribution or reproduction in other forums is permitted, provided the original author(s) and the copyright owner(s) are credited and that the original publication in this journal is cited, in accordance with accepted academic practice. No use, distribution or reproduction is permitted which does not comply with these terms. 